University of Wollongong

Research Online

Faculty of Engineering and Information

Faculty of Engineering and Information

Sciences - Papers: Part A

Sciences

$1-1-2014$

\title{
An adaptively CCA-secure ciphertext-policy attribute-based proxy re- encryption for cloud data sharing
}

Kaitai Liang

City University of Hong Kong

Man $\mathrm{Ho} \mathrm{Au}$

University of Wollongong, aau@uow.edu.au

Willy Susilo

University of Wollongong, wsusilo@uow.edu.au

Duncan Wong

City University of Hong Kong, dwong@uow.edu.au

Guomin Yang

University of Wollongong, gyang@uow.edu.au

See next page for additional authors

Follow this and additional works at: https://ro.uow.edu.au/eispapers

Part of the Engineering Commons, and the Science and Technology Studies Commons

Research Online is the open access institutional repository for the University of Wollongong. For further information contact the UOW Library: research-pubs@uow.edu.au 


\title{
An adaptively CCA-secure ciphertext-policy attribute-based proxy re-encryption for cloud data sharing
}

\begin{abstract}
A Ciphertext-Policy Attribute-Based Proxy Re-Encryption (CP-ABPRE) employs the PRE technology in the attribute-based en- cryption cryptographic setting, in which the proxy is allowed to convert an encryption under an access policy to another encryption under a new access policy. CP-ABPRE is applicable to many real world applications, such as network data sharing. The existing CP-ABPRE systems, how- ever, leave how to achieve adaptive CCA security as an interesting open problem. This paper, for the rst time, proposes a new CP-ABPRE to tackle the problem by integrating the dual system encryption technology with selective proof technique. The new scheme supports any monotonic access structures. Although our scheme is built in the composite order bilinear group, it is proven adaptively CCA secure in the standard model without jeopardizing the expressiveness of access policy.
\end{abstract}

\section{Keywords}

encryption, re, proxy, attribute, policy, ciphertext, data, secure, cloud, cca, adaptively, sharing

Disciplines

Engineering | Science and Technology Studies

\section{Publication Details}

Liang, K., Au, M., Susilo, W., Wong, D. S., Yang, G. \& Yu, Y. (2014). An adaptively CCA-secure ciphertextpolicy attribute-based proxy re-encryption for cloud data sharing. Lecture Notes in Computer Science, 8434 448-461.

\section{Authors}

Kaitai Liang, Man Ho Au, Willy Susilo, Duncan Wong, Guomin Yang, and Yong Yu 


\title{
An Adaptively CCA-Secure Ciphertext-Policy Attribute-Based Proxy Re-Encryption for Cloud Data Sharing
}

\author{
Kaitai Liang ${ }^{1}$, Man Ho $\mathrm{Au}^{2}$, Willy Susilo ${ }^{2 \star}$, Duncan S. Wong ${ }^{1 \star \star}$,

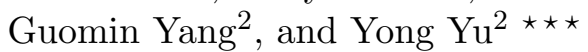 \\ 1 Department of Computer Science, City University of Hong Kong, China \\ kliang4-c@my.cityu.edu.hk, duncan@cityu.edu.hk \\ 2 Centre for Computer and Information Security Research, School of Computer \\ Science and Software Engineering, University of Wollongong, Wollongong, NSW 2522, \\ Australia \\ \{aau, wsusilo, gyang, yyong\}@uow.edu. au
}

\begin{abstract}
A Ciphertext-Policy Attribute-Based Proxy Re-Encryption (CP-ABPRE) employs the PRE technology in the attribute-based encryption cryptographic setting, in which the proxy is allowed to convert an encryption under an access policy to another encryption under a new access policy. CP-ABPRE is applicable to many real world applications, such as network data sharing. The existing CP-ABPRE systems, however, leave how to achieve adaptive CCA security as an interesting open problem. This paper, for the first time, proposes a new CP-ABPRE to tackle the problem by integrating the dual system encryption technology with selective proof technique. The new scheme supports any monotonic access structures. Although our scheme is built in the composite order bilinear group, it is proven adaptively CCA secure in the standard model without jeopardizing the expressiveness of access policy.

Keywords: Ciphertext-Policy Attribute-Based Encryption, CiphertextPolicy Attribute-Based Proxy Re-Encryption, Adaptive Chosen-Ciphertext Security.
\end{abstract}

\section{Introduction}

Attribute-Based Encryption (ABE) [10,21], which is a generalization of Public Key Encryption (PKE), provides flexibility of data sharing for

* W. Susilo is partially supported by the Australian Research Council Linkage Project LP120200052.

${ }^{\star \star}$ D. S. Wong is supported by a grant from the RGC of the HKSAR, China (Project No. CityU 121512).

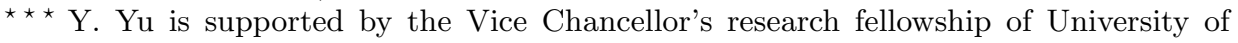
Wollongong and the NSFC of China under Grant 61003232. 
system users such that a data encryptor is allowed to specify some descriptive values $x$ for an encryption and thus, the encryption can be decrypted successfully by a secret key associated with some descriptive values $y$ matching $x$. ABE has many applications, such as audit log applications [10]. It usually has two classifications: Key-Policy ABE (KP-ABE) and Ciphertext-Policy ABE (CP-ABE). In a KP-ABE system, ciphertexts are associated with attribute sets and secret keys are associated with access policies. However, CP-ABE is complementary. This paper deals with the case of $\mathrm{CP}-\mathrm{ABE}$.

In a cloud storage system, a user, say Alice, may encrypt a data under a specified access policy such that other system users satisfying this policy can access the data. She might encrypt her profile under a policy $A P_{1}=$ ("Department: Human Resource" and "Position: Team manager or above") before uploading to the cloud. The system users satisfying $A P_{1}$ then can download the ciphertext from the cloud, and next access the data by using the corresponding secret keys. This data sharing pattern, nonetheless, does not scale well when the policy needs to be updated frequently. Suppose the policy above is updated as $A P_{2}=$ ("Department: Human Resource or Materials Storage" and "Position: Team manager only"), Alice then should generate a new encryption accordingly. If Alice does not back up the data locally, she needs to download the ciphertext so as to recovers the data first. If the access policy is updated $N$ times, Alice needs to construct $N$ new encryptions. This might not be desirable as Alice's workload is linearly in $N$. Besides, if she is off-line or using some resource-limited devices which cannot afford such heavy computational cost, the data sharing might not be handled effectively.

To efficiently share data, we may leverage Proxy Re-Encryption (PRE). PRE is introduced by Mambo and Okamoto [19], and further studied by Blaze, Bleumer and Strauss [5]. It is an interesting extension of PKE providing the delegation of decryption rights. Specifically, it allows a semitrusted proxy to transform a ciphertext intended for Alice into another ciphertext of the same plaintext intended for another system user, say Bob, without revealing knowledge of the secret keys and the underlying plaintext. It is applicable to many network applications, such as secure distributed files systems [1] and email forwarding [5].

To integrate PRE in the ABE cryptographic setting, Liang et al. [16] defined Ciphertext-Policy Attribute-Based PRE (CP-ABPRE), and proposed a concrete $\mathrm{CP}-\mathrm{ABPRE}$ system enabling proxy to transform an encryption under a specified access policy into another encryption under a new access policy. We refer to this special functionality as attribute-based 
re-encryption. By using the technology of CP-ABPRE, Alice can share the data more efficiently. She first generates a re-encryption key from her own attribute set to a new access policy $A P_{2}$, and next uploads the key to the cloud such that the cloud server then can convert the original encryption under $A P_{1}$ to a new encryption under $A P_{2}$. The server, nevertheless, cannot learn the data during the conversion of cipehrtexts.

Although CP-ABPRE explores the applications of PRE, they leave us interesting open problems. All the existing CP-ABPRE schemes in the literature are secure against selective chosen-plaintext attacks (selective CPA) only except [15] which is selective chosen-ciphertext attacks (selective CCA) secure. We state that CPA security might not be sufficient enough in an open network as it only guarantees the secrecy of data which only allows an encryption to be secure against "static" adversaries. Nevertheless, in a real network scenario, there might exist "active" adversaries trying to tamper an encryption in transit and next observing its decryption so as to obtain useful information related to the underlying data. Accordingly, a CP-ABPRE system being secure against CCA is needed as CCA security not only helps the system preclude the above subtle attacks but also enables the system to be further developed and next securely "embedded" to a large protocol/system implementing in arbitrary network environments. In addition, a CP-ABPRE system with selective security, which limits an adversary to choose an attack target before playing security game, might not scale in practice as well. This is so because a realistic adversary can adaptively choose his attack target upon attacking a cryptosystem. Therefore, an adaptively CCA secure CP-ABPRE scheme is needed in most of practical network applications.

The expressiveness of access policy is another crucial factor for a practical CP-ABPRE system. An access policy should be embedded with $A N D, O R$ gates, and even more meaningful expression. For instance, Alice might choose to share her profile with some officials of the same company under the access policy $A P_{3}=$ ("Department : allexcept Human Resource" and "Position : Project head or team manager"). Nevertheless, most of the existing CP-ABPRE schemes only support access policy with $A N D$ gates operating over attributes. This limits their practical use. Thus it is desirable to propose a CP-ABPRE system supporting more expressive access policy.

\subsection{Our Contributions}

This work first formalizes the notion of adaptive CCA security for CPABPRE systems. Compared to the selective CPA security notion, our new 
notion enables an adversary to commit to a target access policy in the challenge phase, and to gain access to re-encryption and decryption oracles additionally. To tackle the open problems mentioned previously, this paper proposes a novel single-hop unidirectional CP-ABPRE system. In addition, the new system supports any monotonic access policy such that system users are allowed to fulfill more flexible delegation of decryption rights. Despite our scheme is built in the composite order bilinear group, it is proven adaptively CCA secure in the standard model by integrating the dual system encryption technology with the selective proof technique.

\subsection{Related Work}

Below we review some ABE systems related to this work. Following the introduction of ABE due to Sahai and Waters [21], Goyal et al. [10] proposed the first KP-ABE system. Later, Bethencourt, Sahai and Waters [4] defined a complementary notion, i.e. CP-ABE. After that there are some CP-ABE schemes (e.g. [7,9,22,2]) that have been proposed. Recently, Waters [23] proposed a deterministic finite automata-based functional encryption where policy is expressed by arbitrary-size regular language.

The aforementioned schemes, nonetheless, are only selective secure (except for [4] being proven in the generic group model). To convert one of the CP-ABE systems [22] to achieve fully security, Lewko et al. [13] leveraged the dual system encryption technology. But their conversion yields some loss of expressiveness. Later, Lewko and Waters [14] introduced a new method to guarantee the expressiveness by employing the selective proof technique into the dual system encryption technology. Inspired by [14,22], this paper focuses on constructing the first CP-ABPRE with adaptive CCA security in the standard model.

Decryption rights delegation is introduced in [19]. Later, Blaze, Bleumer and Strauss [5] defined PRE. PRE can be classified as: unidirectional and bidirectional PRE, and single-hop and multi-hop PRE [1]. This present work deals with the single-hop unidirectional case. Since its introduction many PRE systems have been proposed, e.g., [1,6,12,17,11,24,25,26].

To employ PRE in the context of ABE, Liang et al. [16] defined CPABPRE, and further extended [7] to support proxy re-encryption. Their work provides $A N D$ gates over positive and negative attributes. Luo et al. [18] proposed an extension of [16] supporting policy with $A N D$ gates on multi-valued and negative attributes. To combine ABE with IBE by using PRE technique, Mizuno and Doi [20] proposed a special type of CPABPRE scheme where encryptions in the form of ABE can be converted 
to the ones being decrypted in the context of IBE. The previously introduced systems, however, are selectively CPA secure, and their policies are lack of expressiveness due to supporting $A N D$ gates over attributes only. Thus an adaptively CCA-secure CP-ABPRE scheme with more expressive access policy remains open. This paper deals with this problem.

Below we compare this work with some CP-ABPRE schemes. We let $p$ be the number of attributes used in an access policy, $a$ be the number of attributes embedded in a user's secret key and $u$ be the total number of attributes used in the system. In the worst case, an access policy and a user's secret key might be embedded with all system attributes, that is $p=a=u$. Thus we have $p, a \leq u$. We use $c_{e}$ and $c_{p}$ to denote the computational cost of an exponentiation and a bilinear pairing. To the best of our knowledge, our scheme is the first to achieve adaptive CCA security, and to support any monotonic access formula.

Table 1. Comparison with $[16,18,20]$

\begin{tabular}{|c|c|c|c|c|c|}
\hline \multirow{2}{*}{ Schemes } & \multicolumn{2}{|c|}{ Public/Secret Ciphertext } & \multirow{2}{*}{$\begin{array}{l}\text { Re-Encryption } \\
\text { Cost }\end{array}$} & \multirow{2}{*}{$\begin{array}{r}\text { Adaptive } \\
\text { Security }\end{array}$} & \multirow{2}{*}{$\begin{array}{c}\text { CCA } \\
\text { Security }\end{array}$} \\
\hline & Key Size & Size & & & \\
\hline [16] & $\mathcal{O}(u) / \mathcal{O}(u)$ & $\mathcal{O}(u)$ & $\mathcal{O}(u) \cdot c_{p}$ & $x$ & $\bar{x}$ \\
\hline$[18]$ & $\mathcal{O}\left(u^{2}\right) / \mathcal{O}(u)$ & $\mathcal{O}(u)$ & $\mathcal{O}(u) \cdot c_{p}$ & 0 & X \\
\hline$[20]$ & $\mathcal{O}(u) / \mathcal{O}(u)$ & $\mathcal{O}(u)$ & $\mathcal{O}(1) \cdot c_{e}+\mathcal{O}(u) \cdot c_{p}$ & X & x \\
\hline Ours & $\mathcal{O}(u) / \mathcal{O}(a)$ & $\mathcal{O}(p)$ & $\mathcal{O}(a) \cdot c_{e}+\mathcal{O}(a) \cdot c_{p}$ & $\checkmark$ & $\sqrt{ }$ \\
\hline
\end{tabular}

\section{Definitions and Security Models}

We review the definition of CP-ABPRE systems, and next define the adaptive CCA security notion. Due to limited space we refer the reader to [22] for the details of access structure and Linear Secret Sharing Schemes.

\subsection{Definition of CP-ABPRE}

We review the definition of single-hop unidirectional CP-ABPRE $[16,18]$.

Definition 1. A Single-Hop Unidirectional Ciphertext-Policy AttributeBased Proxy Re-Encryption (CP-ABPRE) scheme consists of the following algorithms: 
1. $($ param,$m s k) \leftarrow \operatorname{Setup}\left(1^{k}, \mathcal{U}\right)$ : on input a security parameter $k \in \mathbb{N}$ and an attribute universe $\mathcal{U}$, output the public parameters param and a master secret key msk.

2. $s k_{S} \leftarrow K e y G e n($ param, $m s k, S)$ : on input param, msk and an attribute set $S$ describing the key, output a secret key $s k_{S}$ for $S$.

3. $r k_{S \rightarrow\left(A^{\prime}, \rho^{\prime}\right)} \leftarrow$ ReKeyGen(param, $\left.s k_{S},\left(A^{\prime}, \rho^{\prime}\right)\right)$ : on input param, sk $k_{S}$, and an access structure $\left(A^{\prime}, \rho^{\prime}\right)$ for attributes over $\mathcal{U}$, output a reencryption key $r k_{S \rightarrow\left(A^{\prime}, \rho^{\prime}\right)}$ which can be used to transform a ciphertext under $(A, \rho)$ to another ciphertext under $\left(A^{\prime}, \rho^{\prime}\right)$, where $S \models(A, \rho)$, $S \not \models\left(A^{\prime}, \rho^{\prime}\right),(A, \rho)$ and $\left(A^{\prime}, \rho^{\prime}\right)$ are two disjoint access structures. Note by two disjoint access structures we mean for any attribute $x$ satisfies $(A, \rho), x$ does not satisfy $\left(A^{\prime}, \rho^{\prime}\right)$.

4. $C \leftarrow$ Encrypt $($ param, $(A, \rho), m)$ : on input param, $(A, \rho)$, and a message $m \in\{0,1\}^{k}$, output an original ciphertext $C$ which can be further re-encrypted. Note $(A, \rho)$ is implicitly included in the ciphertext.

5. $C_{R} \leftarrow \operatorname{ReEnc}\left(\right.$ param, $\left.r k_{S \rightarrow\left(A^{\prime}, \rho^{\prime}\right)}, C\right)$ : on input param, $r k_{S \rightarrow\left(A^{\prime}, \rho^{\prime}\right)}$, and a $C$ under $(A, \rho)$, output a re-encrypted ciphertext $C_{R}$ under $\left(A^{\prime}, \rho^{\prime}\right)$ if $S=(A, \rho)$ or a symbol $\perp$ indicating either $C$ is invalid or $S \not \models(A, \rho)$. Note $C_{R}$ cannot be further re-encrypted.

6. $m \leftarrow \operatorname{Dec}\left(\right.$ param, $\left.s k_{S}, C\right)$ : on input param, $s k_{S}$, and a $C$ under $(A, \rho)$, output a message $m$ if $S \models(A, \rho)$ or a symbol $\perp$ indicating either $C$ is invalid or $S \not \models(A, \rho)$.

7. $m \leftarrow \operatorname{Dec}_{R}\left(\right.$ param, $\left.s k_{S}, C_{R}\right)$ : on input param, sk $k_{S}$, and a $C_{R}$ under $(A, \rho)$, output a message $m$ if $S \models(A, \rho)$ or a symbol $\perp$ indicating either $C_{R}$ is invalid or $S \not \models(M, \rho)$.

\subsection{Security Models}

Definition 2. A single-hop unidirectional CP-ABPRE scheme is IND$C C A$ secure at original ciphertext if no Probabilistic Polynomial Time (PPT) adversary $\mathcal{A}$ can win the game below with non-negligible advantage. Below $\mathcal{C}$ is the game challenger.

1. Setup. $\mathcal{C}$ runs $\operatorname{Setup}\left(1^{k}, \mathcal{U}\right)$ and sends param to $\mathcal{A}$.

2. Phase 1.

(a) Secret key extraction oracle $\mathcal{O}_{s k}(S)$ : on input an attribute set $S$, $\mathcal{C}$ runs $s k_{S} \leftarrow$ KeyGen (param, msk, $\left.S\right)$ and returns sk $k_{S}$ to $\mathcal{A}$.

(b) Re-encryption key extraction oracle $\mathcal{O}_{r k}\left(S,\left(A^{\prime}, \rho^{\prime}\right)\right)$ : on input $S$, and an access structure $\left(A^{\prime}, \rho^{\prime}\right), \mathcal{C}$ outputs $r k_{S \rightarrow\left(A^{\prime}, \rho^{\prime}\right)} \leftarrow$ ReKeyGen $\left(\right.$ param, $\left.k_{S},\left(A^{\prime}, \rho^{\prime}\right)\right)$, where $s k_{S} \leftarrow \operatorname{KeyGen}($ param, msk, $S)$. 
(c) Re-encryption oracle $\mathcal{O}_{r e}\left(S,\left(A^{\prime}, \rho^{\prime}\right), C\right)$ : on input $S,\left(A^{\prime}, \rho^{\prime}\right)$, an original ciphertext $C$ under $(A, \rho), \mathcal{C}$ outputs $C_{R} \leftarrow \operatorname{ReEnc}($ param, $\left.r k_{S \rightarrow\left(A^{\prime}, \rho^{\prime}\right)}, C\right)$, where $r k_{S \rightarrow\left(A^{\prime}, \rho^{\prime}\right)} \leftarrow \operatorname{ReKeyGen}\left(\right.$ param, $s k_{S},\left(A^{\prime}\right.$, $\left.\left.\rho^{\prime}\right)\right), s k_{S} \leftarrow$ KeyGen (param, msk, $\left.S\right)$ and $S \models(A, \rho)$.

(d) Original ciphertext decryption oracle $\mathcal{O}_{\text {dec }}(S, C)$ : on input $S$ and a $C$ under $(A, \rho), \mathcal{C}$ returns $m \leftarrow \operatorname{Dec}\left(\right.$ param, $\left.s k_{S}, C\right)$ to $\mathcal{A}$, where $s k_{S} \leftarrow$ KeyGen $($ param, msk, $S)$ and $S \models(A, \rho)$.

(e) Re-encrypted ciphertext decryption oracle $\mathcal{O}_{\operatorname{dec}_{R}}\left(S, C_{R}\right)$ : on input $S$ and $a C_{R}$ under $(A, \rho), \mathcal{C}$ returns $m \leftarrow D e c_{R}\left(\right.$ param, $\left.s k_{S}, C_{R}\right)$, where $s k_{S} \leftarrow \operatorname{KeyGen}($ param, msk, $S)$ and $S \models(A, \rho)$.

If ciphertexts issued to $\mathcal{O}_{\text {re }}, \mathcal{O}_{\text {dec }}$ and $\mathcal{O}_{\text {dec }_{R}}$ are invalid, outputs $\perp$.

3. Challenge. $\mathcal{A}$ outputs two equal length messages $m_{0}$ and $m_{1}$, and a challenge access structure $\left(A^{*}, \rho^{*}\right)$ to $\mathcal{C}$. If the following queries

$$
\begin{aligned}
& \mathcal{O}_{s k}(S) \text { for any } S \models\left(A^{*}, \rho^{*}\right) \text {; and } \\
& \mathcal{O}_{r k}\left(S,\left(A^{\prime}, \rho^{\prime}\right)\right) \text { for any } S \models\left(A^{*}, \rho^{*}\right), \mathcal{O}_{s k}\left(S^{\prime}\right) \text { for any } S^{\prime} \models\left(A^{\prime}, \rho^{\prime}\right)
\end{aligned}
$$

are never made, $\mathcal{C}$ returns $C^{*}=\operatorname{Encrypt}\left(\operatorname{param},\left(A^{*}, \rho^{*}\right), m_{b}\right)$ to $\mathcal{A}$, where $b \in_{R}\{0,1\}$.

4. Phase 2. $\mathcal{A}$ continues making queries except the followings:

(a) $\mathcal{O}_{s k}(S)$ for any $S \models\left(A^{*}, \rho^{*}\right)$;

(b) $\mathcal{O}_{r k}\left(S,\left(A^{\prime}, \rho^{\prime}\right)\right)$ for any $S \models\left(A^{*}, \rho^{*}\right)$, and $\mathcal{O}_{s k}\left(S^{\prime}\right)$ for any $S^{\prime} \models$ $\left(A^{\prime}, \rho^{\prime}\right)$;

(c) $\mathcal{O}_{r e}\left(S,\left(A^{\prime}, \rho^{\prime}\right), C^{*}\right)$ for any $S \models\left(A^{*}, \rho^{*}\right)$, and $\mathcal{O}_{s k}\left(S^{\prime}\right)$ for any $S^{\prime} \models$ $\left(A^{\prime}, \rho^{\prime}\right)$;

(d) $\mathcal{O}_{\text {dec }}\left(S, C^{*}\right)$ for any $S=\left(A^{*}, \rho^{*}\right)$; and

(e) $\mathcal{O}_{\operatorname{dec}_{R}}\left(S, C_{R}\right)$ for any $C_{R}$ under $(A, \rho), S \models(A, \rho)$, where $C_{R}$ is a derivative of $C^{*}$. As of [6], the derivative of $C^{*}$ is defined as:

i. $C^{*}$ is a derivative of itself.

ii. If $\mathcal{A}$ has issued a re-encryption key query on $\left(S^{*},\left(A^{\prime}, \rho^{\prime}\right)\right)$ to get $r k_{S^{*} \rightarrow\left(A^{\prime}, \rho^{\prime}\right)}$, obtained $C_{R} \leftarrow \operatorname{Re} \operatorname{Enc}\left(\right.$ param, $r k_{S^{*} \rightarrow\left(A^{\prime}, \rho^{\prime}\right)}$, $\left.C^{*}\right)$ such that $\operatorname{Dec}_{R}\left(\right.$ param, sk $\left.k_{S^{\prime}}, C_{R}\right) \in\left\{m_{0}, m_{1}\right\}$, then $C_{R}$ is a derivative of $C^{*}$, where $S^{*} \models\left(A^{*}, \rho^{*}\right)$ and $S^{\prime} \models\left(A^{\prime}, \rho^{\prime}\right)$.

iii. If $\mathcal{A}$ has issued a re-encryption query on $\left(S,\left(A^{\prime}, \rho^{\prime}\right), C^{*}\right)$ and obtained the re-encrypted ciphertext $C_{R}$, then $C_{R}$ is a derivative of $C^{*}$, where $S \models\left(A^{*}, \rho^{*}\right)$.

5. Guess. $\mathcal{A}$ outputs a guess bit $b^{\prime} \in\{0,1\}$. If $b^{\prime}=b, \mathcal{A}$ wins.

$\mathcal{A}$ 's advantage is defined as $A d v_{C P-A B P R E, \mathcal{A}}^{I N D-C C A-O r}\left(1^{k}, \mathcal{U}\right)=\left|\operatorname{Pr}\left[b^{\prime}=b\right]-\frac{1}{2}\right|$.

Definition 3. A single-hop unidirectional CP-ABPRE scheme is IND$C C A$ secure at re-encrypted ciphertext if the advantage $A d v_{C P-A B P R E, A}^{I N D-C C A-R e}\left(1^{k}\right.$, 
$\mathcal{U})$ is negligible for any PPT adversary $\mathcal{A}$ in the following experiment. Set $\mathcal{O}=\left\{\mathcal{O}_{s k}, \mathcal{O}_{r k}, \mathcal{O}_{d e c}, \mathcal{O}_{\operatorname{dec}_{R}}\right\}$

$$
\begin{aligned}
& A d v_{C P-A B P R E, \mathcal{A}}^{I N D-C C A-R e}\left(1^{k}, \mathcal{U}\right)=\mid \operatorname{Pr}\left[b^{\prime}=b:(\text { param }, \text { msk }) \leftarrow \operatorname{Setup}\left(1^{k}, \mathcal{U}\right) ;\right. \\
& \left(m_{0}, m_{1},\left(A^{*}, \rho^{*}\right),(A, \rho)\right) \leftarrow \mathcal{A}^{\mathcal{O}}(\text { param }) ; b \in_{R}\{0,1\} ; \\
& \left.C_{R}^{*} \leftarrow \operatorname{ReEnc}\left(\text { param }, r k_{S \rightarrow\left(A^{*}, \rho^{*}\right)}, C\right) ; b^{\prime} \leftarrow A^{\mathcal{O}}\left(C_{R}^{*}\right)\right]-\frac{1}{2} \mid,
\end{aligned}
$$

where $(A, \rho)$ and $\left(A^{*}, \rho^{*}\right)$ are disjoint, $\left(A^{*}, \rho^{*}\right)$ is the challenge access structure, $S \models(A, \rho), r k_{S \rightarrow\left(A^{*}, \rho^{*}\right)} \leftarrow \operatorname{ReKeyGen}\left(\operatorname{param}, s k_{S},\left(A^{*}, \rho^{*}\right)\right)$, $C \leftarrow \operatorname{Encrypt}\left(\right.$ param $\left.,(A, \rho), m_{b}\right), \mathcal{O}_{s k}, \mathcal{O}_{r k}, \mathcal{O}_{d e c}, \mathcal{O}_{d e c_{R}}$ are the oracles defined in Definition 2. However, these oracles are restricted by the following constraints. For $\mathcal{O}_{s k}$, any query $S=\left(A^{*}, \rho^{*}\right)$ is rejected. There is no restriction to $\mathcal{O}_{r k}$ and $\mathcal{O}_{\text {dec }}$ (note invalid ciphertexts issued to $\mathcal{O}_{\text {dec }}$ are rejected). If $\mathcal{A}$ queries to $\mathcal{O}_{\text {dec }_{R}}$ on either $\left(S, C_{R}^{*}\right)$ in which $S \models\left(A^{*}, \rho^{*}\right)$ or any invalid re-encrypted ciphertext, the oracle outputs $\perp$.

Remarks. Definition 3 implies collusion resistance. If $\mathcal{A}$ can compromise $s k_{S^{*}}$ from either $r k_{S^{*} \rightarrow(A, \rho)}$ or $r k_{S \rightarrow\left(A^{*}, \rho^{*}\right)}, \mathcal{A}$ wins the game with nonnegligible probability, where $S \models(A, \rho), S^{*}=\left(A^{*}, \rho^{*}\right)$ and $s k_{S}$ is given.

\section{An Adaptively CCA-Secure CP-ABPRE}

\subsection{Construction}

Due to limited space we review composite order bilinear groups, complexity assumptions, and one-time symmetric encryption in Appendix A.

1. $\operatorname{Setup}\left(1^{k}, \mathcal{U}\right)$. Run $\left(N, \mathbb{G}, \mathbb{G}_{T}, e\right) \leftarrow \mathcal{G}\left(1^{k}\right)$, where $N=p_{1} p_{2} p_{3}$ is the order of group $\mathbb{G}$ and $p_{1}, p_{2}, p_{3}$ are distinct primes. Let $\mathbb{G}_{p_{i}}$ denote the subgroup of order $p_{i}$ in group $\mathbb{G}$. Choose $a, \alpha, \kappa, \beta, \epsilon \in \in_{R} \mathbb{Z}_{N}, g, \hat{g}_{1} \in R$ $\mathbb{G}_{p_{1}}$, two Target Collision Resistance hash functions [8] $T C R_{1}: \mathbb{G}_{T} \rightarrow$ $\mathbb{Z}_{N}, T C R_{2}: \mathbb{G}_{T} \rightarrow\{0,1\}^{\text {poly }\left(1^{k}\right)}$, a CCA-secure one-time symmetric encryption system $S Y M$ and a strongly existential unforgeable onetime signature system [3] OTS. For each attribute $i \in \mathcal{U}$, choose $h_{i} \in R \mathbb{Z}_{N}$. The param is $\left(N, g, \hat{g}_{1}, g^{a}, g^{\kappa}, g^{\beta}, g^{\epsilon}, e(g, g)^{\alpha}, \forall i \in \mathcal{U} H_{i}=\right.$ $\left.g^{h_{i}}, T C R_{1}, T C R_{2}, S Y M, O T S\right)$, and the $m s k$ is $\left(g^{\alpha}, g_{3}\right)$, where $g_{3}$ is a generator of $\mathbb{G}_{p_{3}}$.

2. KeyGen $($ param, msk, $S)$. Choose $t, u \in \in_{R} \mathbb{Z}_{N}, R, R^{\prime}, R^{\prime \prime},\left\{R_{i}\right\}_{i \in S} \in_{R}$ $\mathbb{G}_{p_{3}}$, and set the secret key $s k_{S}$ as

$$
\left(S, K=g^{\alpha} g^{a t} g^{\kappa u} R, K^{\prime}=g^{u} R^{\prime}, K^{\prime \prime}=g^{t} R^{\prime \prime}, \forall i \in S K_{i}=H_{i}^{t} R_{i}\right) .
$$


3. $\operatorname{Encrypt}($ param $,(A, \rho), m)$. Given an LSSS access structure $(A, \rho)$ and a message $m \in \mathbb{G}_{T}$ in which $A$ is an $l \times n$ matrix and $\rho$ is a map from each row $A_{j}$ to an attribute $\rho(j)$,

(a) Choose a random vector $v=\left(s, v_{2}, \ldots, v_{n}\right) \in_{R} \mathbb{Z}_{N}^{n}$.

(b) For each $A_{j}$, choose $r_{j} \in R \mathbb{Z}_{N}$, run $(s s k, s v k) \leftarrow$ OTS.KeyGen $\left(1^{k}\right)$ and set

$$
\begin{aligned}
& B_{0}=m \cdot e(g, g)^{\alpha s}, B_{1}=g^{s}, B_{2}=\left(g^{\kappa}\right)^{s}, B_{3}=\left(\hat{g}_{1}^{s v k} g^{\beta}\right)^{s}, B_{4}=\left(g^{\epsilon}\right)^{s}, \\
& \forall j \in[1, l]\left(C_{j}=\left(g^{a}\right)^{A_{j} v} H_{\rho(j)}{ }^{-r_{j}}, D_{j}=g^{r_{j}}\right), \\
& E=O T S . S i g n\left(s s k,\left(B_{0}, B_{1}, B_{3}, \forall j \in[1, l]\left(C_{j}, D_{j}\right)\right)\right) .
\end{aligned}
$$

(c) Output $C=\left(s v k, B_{0}, B_{1}, B_{2}, B_{3}, B_{4}, \forall j \in[1, l]\left(C_{j}, D_{j}\right), E\right)$. Note $\{\rho(j) \mid 1 \leq j \leq l\}$ are the attributes used in $(A, \rho)$.

4. ReKeyGen $\left(\right.$ param, $\left.s k_{S},\left(A^{\prime}, \rho^{\prime}\right)\right)$. Given $s k_{S}=\left(S, K, K^{\prime}, K^{\prime \prime}, \forall i \in\right.$ $\left.S K_{i}\right)$ and an LSSS access structure $\left(A^{\prime}, \rho^{\prime}\right)$,

(a) Choose $\theta_{1}, \theta_{2}, \theta_{3} \in_{R} \mathbb{Z}_{N}, \delta \in_{R} \mathbb{G}_{T}$, set $r k_{1}=\left(K g^{\kappa \theta_{1}} g^{a \theta_{2}}\right)^{T C R_{1}(\delta)} g^{\epsilon \theta_{3}}$, $r k_{2}=\left(K^{\prime} g^{\theta_{1}}\right)^{T C R_{1}(\delta)}, r k_{3}=\left(K^{\prime \prime} g^{\theta_{2}}\right)^{T C R_{1}(\delta)}, r k_{4}=g^{\theta_{3}}, \forall i \in S$ $r k_{5, i}=\left(K_{i} H_{i}^{\theta_{2}}\right)^{T C R_{1}(\delta)}$.

(b) Choose a random vector $v^{(r k)}=\left(s^{(r k)}, v_{2}^{(r k)}, \ldots, v_{n}^{(r k)}\right) \in_{R} \mathbb{Z}_{N}^{n}$. For each row $A_{j}^{\prime}$ of $A^{\prime}$, choose $r_{j}^{(r k)} \in_{R} \mathbb{Z}_{N}$, run $\left(s s k^{(r k)}, s v k^{(r k)}\right) \leftarrow$ OTS.KeyGen $\left(1^{k}\right)$ and set $r k_{6}$ as

$$
\begin{aligned}
& s v k^{(r k)}, B_{0}^{(r k)}=\delta \cdot e(g, g)^{\alpha s^{(r k)}}, B_{1}^{(r k)}=g^{s^{(r k)}}, B_{2}^{(r k)}=\left(g^{\kappa}\right)^{s^{(r k)}}, \\
& B_{3}^{(r k)}=\left(\hat{g}_{1}^{s v k^{(r k)}} g^{\beta}\right)^{s^{(r k)}}, \forall j \in[1, l]\left(C_{j}^{(r k)}=\left(g^{a}\right)^{A_{j}^{\prime} v^{(r k)}} H_{\rho^{\prime}(j)}{ }^{-r_{j}^{(r k)}},\right. \\
& \left.D_{j}^{(r k)}=g_{j}^{r^{(r k)}}\right), E^{(r k)}=O T S . S i g n\left(s s k^{(r k)},\left(B_{0}^{(r k)}, B_{1}^{(r k)}, B_{3}^{(r k)},\right.\right. \\
& \left.\left.\forall j \in[1, l]\left(C_{j}^{(r k)}, D_{j}^{(r k)}\right)\right)\right) .
\end{aligned}
$$

(c) Output $r k_{S \rightarrow\left(A^{\prime}, \rho^{\prime}\right)}=\left(r k_{1}, r k_{2}, r k_{3}, r k_{4}, \forall i \in S r k_{5, i}, r k_{6}\right)$.

5. ReEnc $\left(\right.$ param, $\left.r k_{S \rightarrow\left(A^{\prime}, \rho^{\prime}\right)}, C\right)$. Parse the original ciphertext $C$ under $(A, \rho)$ as $\left(s v k, B_{0}, B_{1}, B_{2}, B_{3}, B_{4}, \forall j \in[1, l]\left(C_{j}, D_{j}\right), E\right)$, and the re-encryption key $r k_{S \rightarrow\left(A^{\prime}, \rho^{\prime}\right)}$ as $\left(r k_{1}, r k_{2}, r k_{3}, r k_{4}, \forall i \in S r k_{5, i}, r k_{6}\right)$.

(a) Check the validity of the original ciphertext $C$ as

$$
\begin{aligned}
& e\left(B_{1}, g^{\kappa}\right) \stackrel{?}{=} e\left(B_{2}, g\right), e\left(B_{1}, \hat{g}_{1}^{s v k} g^{\beta}\right) \stackrel{?}{=} e\left(B_{3}, g\right), e\left(B_{1}, g^{\epsilon}\right) \stackrel{?}{=} e\left(B_{4}, g\right), \\
& e\left(\prod_{\rho(j) \in S} C_{j}^{w_{j}}, g\right) \stackrel{?}{=} e\left(B_{1}, g^{a}\right) \cdot \prod_{\rho(j) \in S}\left(e\left(D_{j}^{-1}, H_{\rho(j)}^{w_{j}}\right)\right), S \stackrel{?}{=}(A, \rho), \\
& \text { OTS.Verify }\left(\operatorname{svk},\left(E,\left(B_{0}, B_{1}, B_{3}, \forall j \in[1, l]\left(C_{j}, D_{j}\right)\right)\right)\right) \stackrel{?}{=} 1, \quad(1)
\end{aligned}
$$

where $w_{j}$ are chosen by the proxy so that $\sum_{\rho(j) \in S} w_{j} A_{j}=(1,0$, ..., 0). If Eq. (1) does not hold, output $\perp$. Otherwise, proceed. 
(b) Compute $F=\frac{e\left(B_{1}, r k_{1}\right) e\left(B_{2}, r k_{2}\right)^{-1} e\left(B_{4}, r k_{4}\right)^{-1}}{\left(\prod_{\rho(j) \in S}\left(e\left(C_{j}, r k_{3}\right) e\left(D_{j}, r k_{5, j}\right)\right)^{w_{j}}\right)}$, run $\sigma_{1}=S Y M \cdot \operatorname{Enc}($ $\left.T C R_{2}(k e y), G\right)$, where $G=\left(C\left\|r k_{6}\right\| F\right)$ and key $\in_{R} \mathbb{G}_{T}$.

(c) Choose a random vector $v^{(r e)}=\left(s^{(r e)}, v_{2}^{(r e)}, \ldots, v_{n}^{(r e)}\right) \in_{R} \mathbb{Z}_{N}^{n}$. For each row $A_{j}^{\prime}$ of $A^{\prime}$, choose $r_{j}^{(r e)} \in_{R} \mathbb{Z}_{N}$, run $\left(s s k^{(r e)}, s v k^{(r e)}\right) \leftarrow$ OTS.KeyGen $\left(1^{k}\right)$ and set $\sigma_{2}$ as

$$
\begin{aligned}
& s v k^{(r e)}, B_{0}^{(r e)}=k e y \cdot e(g, g)^{\alpha s^{(r e)}}, B_{1}^{(r e)}=g^{s^{(r e)}}, B_{2}^{(r e)}=\left(g^{\kappa}\right)^{s^{(r e)}}, \\
& B_{3}^{(r e)}=\left(\hat{g}_{1}^{s v k^{(r e)}} g^{\beta}\right)^{s^{(r e)}}, \forall j \in[1, l]\left(C_{j}^{(r e)}=\left(g^{a}\right)^{A_{j}^{\prime} v^{(r e)}} H_{\rho^{\prime}(j)}{ }^{-r_{j}^{(r e)}},\right. \\
& \left.D_{j}^{(r e)}=g^{r^{(r e)}}\right), E^{(r e)}=O T S . S i g n\left(s s k^{(r e)},\left(B_{0}^{(r e)}, B_{1}^{(r e)}, B_{3}^{(r e)},\right.\right. \\
& \left.\left.\forall j \in[1, l]\left(C_{j}^{(r e)}, D_{j}^{(r e)}\right)\right)\right) .
\end{aligned}
$$

(d) Output $C_{R}=\left(\sigma_{1}, \sigma_{2}\right)$ under $\left(A^{\prime}, \rho^{\prime}\right)$.

6. Dec $\left(\right.$ param, $\left.s k_{S}, C\right)$. Parse the original ciphertext $C$ under $(A, \rho)$ as (svk, $\left.B_{0}, B_{1}, B_{2}, B_{3}, B_{4}, \forall j \in[1, l]\left(C_{j}, D_{j}\right), E\right)$, and the secret key $s k_{S}$ as $\left(S, K, K^{\prime}, K^{\prime \prime}, \forall i \in S K_{i}\right)$. The decryption algorithm chooses a set of constants $w_{j} \in_{R} \mathbb{Z}_{N}$ such that $\sum_{\rho(j) \in S} w_{j} A_{j}=(1,0, \ldots, 0)$, and next recovers the message as follows.

(a) If Eq. (1) does not hold, output $\perp$. Otherwise, proceed.

(b) Compute $e\left(B_{1}, K\right) e\left(B_{2}, K^{\prime}\right)^{-1} /\left(\prod_{\rho(j) \in S}\left(e\left(C_{j}, K^{\prime \prime}\right) e\left(D_{j}, K_{\rho(j)}\right)\right)^{w_{j}}\right)$ $=e(g, g)^{\alpha s}$, and output the message $m=B_{0} / e(g, g)^{\alpha s}$.

7. $\operatorname{Dec}_{R}\left(\right.$ param, $\left.s k_{S}, C_{R}\right)$. Parse the re-encrypted ciphertext $C_{R}$ under $\left(A^{\prime}, \rho^{\prime}\right)$ as $\left(\sigma_{1}, \sigma_{2}\right)$, and the secret key $s k_{S}$ as $\left(S, K, K^{\prime}, K^{\prime \prime}, \forall i \in S K_{i}\right)$.

(a) Check the validity of $\sigma_{2}$ as

$$
\begin{aligned}
& e\left(B_{1}^{(r e)}, g^{\kappa}\right) \stackrel{?}{=} e\left(B_{2}^{(r e)}, g\right), e\left(B_{1}^{(r e)}, \hat{g}_{1}^{s v k^{(r e)}} g^{\beta}\right) \stackrel{?}{=} e\left(B_{3}^{(r e)}, g\right), \\
& e\left(\prod_{\rho^{\prime}(j) \in S}\left(C_{j}^{(r e)}\right)^{w_{j}^{(r e)}}, g\right) \stackrel{?}{=} e\left(B_{1}^{(r e)}, g^{a}\right) \cdot \prod_{\rho^{\prime}(j) \in S}\left(e\left(\left(D_{j}^{(r e)}\right)^{-1}, H_{\rho^{\prime}(j)}^{w_{j}^{(r e)}}\right)\right), \\
& \text { OTS.Verify }\left(\operatorname{svk} k^{(r e)},\left(E^{(r e)},\left(B_{0}^{(r e)}, B_{1}^{(r e)}, B_{3}^{(r e)},\right.\right.\right. \\
& \left.\left.\left.\forall j \in[1, l]\left(C_{j}^{(r e)}, D_{j}^{(r e)}\right)\right)\right)\right) \stackrel{?}{=} 1, S \stackrel{?}{=}\left(A^{\prime}, \rho^{\prime}\right)
\end{aligned}
$$

where $w_{j}^{(r e)}$ are chosen by the decryptor so that $\sum_{\rho^{\prime}(j) \in S} w_{j}^{(r e)} A_{j}^{\prime}=$ $(1,0, \ldots, 0)$. If Eq. (2) does not hold, output $\perp$. Otherwise, proceed. (b) Compute $e\left(B_{1}^{(r e)}, K\right) e\left(B_{2}^{(r e)}, K^{\prime}\right)^{-1} /\left(\prod_{\rho^{\prime}(j) \in S}\left(e\left(C_{j}^{(r e)}, K^{\prime \prime}\right) e\left(D_{j}^{(r e)}\right.\right.\right.$, $\left.\left.\left.K_{\rho^{\prime}(j)}\right)\right)^{w_{j}^{(r e)}}\right)=e(g, g)^{\alpha s^{(r e)}}$, and output key $=B_{0}^{(r e)} / e(g, g)^{\alpha s^{(r e)}}$.

(c) Run $G=S Y M \cdot \operatorname{Dec}\left(T C R_{2}(k e y), \sigma_{1}\right)$. 
(d) Parse $G$ as $\left(C, r k_{6}, F\right)$. If either Eq. (1) or the following verification for $r k_{6}$ does not hold, output $\perp$. Otherwise, proceed.

$$
\begin{aligned}
& e\left(B_{1}^{(r k)}, g^{\kappa}\right) \stackrel{?}{=} e\left(B_{2}^{(r k)}, g\right), e\left(B_{1}^{(r k)}, \hat{g}_{1}^{s v k^{(r k)}} g^{\beta}\right) \stackrel{?}{=} e\left(B_{3}^{(r k)}, g\right) \\
& e\left(\prod_{\rho^{\prime}(j) \in S}\left(C_{j}^{(r k)}\right)^{w_{j}^{(r k)}}, g\right) \stackrel{?}{=} e\left(B_{1}^{(r k)}, g^{a}\right) \cdot \prod_{\rho^{\prime}(j) \in S}\left(e\left(\left(D_{j}^{(r k)}\right)^{-1}, H_{\rho^{\prime}(j)}^{w_{j}^{(r k)}}\right)\right) \\
& \text { OTS.Verify }\left(\operatorname{svk} k^{(r k)},\left(E^{(r k)},\left(B_{0}^{(r k)}, B_{1}^{(r k)}, B_{3}^{(r k)}\right.\right.\right. \\
& \left.\left.\left.\forall j \in[1, l]\left(C_{j}^{(r k)}, D_{j}^{(r k)}\right)\right)\right)\right) \stackrel{?}{=} 1, S \stackrel{?}{=}\left(A^{\prime}, \rho^{\prime}\right)
\end{aligned}
$$

where $w_{j}^{(r k)}$ are chosen by the decryptor so that $\sum_{\rho^{\prime}(j) \in S} w_{j}^{(r k)} A_{j}^{\prime}=$ $(1,0, \ldots, 0)$.

(e) Compute $e\left(B_{1}^{(r k)}, K\right) e\left(B_{2}^{(r k)}, K^{\prime}\right)^{-1} /\left(\prod_{\rho^{\prime}(j) \in S}\left(e\left(C_{j}^{(r k)}, K^{\prime \prime}\right) e\left(D_{j}^{(r k)}\right.\right.\right.$, $\left.\left.\left.K_{\rho^{\prime}(j)}\right)\right)^{w_{j}^{(r k)}}\right)=e(g, g)^{\alpha s^{(r k)}}$, and then $B_{0}^{(r k)} / e(g, g)^{\alpha s^{(r k)}}=\delta$. Compute $F^{T C R_{1}(\delta)^{-1}}=e(g, g)^{\alpha s}$, and finally output $m=B_{0} / e(g, g)^{\alpha s}$.

\subsection{Security Analysis}

Theorem 1. Suppose Assumption 1, the general subgroup decision assumption, the three party Diffie-Hellman assumption in a subgroup, and the source q-parallel BDHE assumption in a subgroup hold, SYM is a $C C A$-secure one-time symmetric encryption, OTS is a strongly existential unforgeable one-time signature, and $T C R_{1}, T C R_{2}$ are the $T C R$ hash functions, our system is IND-CCA secure in the standard model.

We prove our scheme by following [14]. Due to limited space, we present our construction for semi-functional ciphertexts and semi-functional keys in the full version.

We will prove Theorem 1 in a hybrid argument over a sequence of games. We let the total number of queries be $q=q_{s k}+q_{r k}+q_{r e}+q_{d e c}$, where $q_{s k}, q_{r k}, q_{r e}, q_{d e c}$ denote the number of the secret key, re-encryption key, re-encryption and decryption queries, respectively. Game real is the first game that is the IND-CCA security game for CP-ABPRE systems in which the challenge ciphertext (original ciphertext/re-encrypted ciphertext) is normal. Here, $\mathcal{C}$ will use normal secret keys to respond secret key extraction queries. Besides, $\mathcal{C}$ will first generate normal secret keys, and next leverage these keys to respond the re-encryption key, re-encryption and decryption queries, namely, the re-encryption keys, re-encryption results and decryption results are indirectly computed from the normal 
secret keys. Game 0 is the second game which is identical to Game real except that the challenge ciphertext is semi-functional.

Hereafter by "keys" (resp. "key") we mean the secret key(s) (constructed by $\mathcal{C}$ ) used to respond the secret key extraction, re-encryption key extraction, re-encryption and decryption queries. In the following, we will convert the "keys" to be semi-functional one by one. But for clarity we first turn the "keys" for the secret key extraction queries, and then convert the "keys" for the re-encryption key queries, the re-encryption queries and the decryption queries in sequence. Besides, $\mathcal{A}$ issues one query in each of the following games. We define Game $_{i}$ as follows, where $i \in[1, q]$. We let $j_{\tau} \in\left[1, q_{\tau}\right]$, where $\tau \in\{s k, r k, r e, d e c\}$. In $G_{a m e} j_{\tau}$ we define two sub-games $\operatorname{Game}_{j_{\tau}}^{N}$ and $\operatorname{Game}_{j_{\tau}}^{T}$ in which the challenge ciphertext is semifunctional. In $\operatorname{Game}_{j_{\tau}}^{N}$ the first $(j-1)_{\tau}$ "keys" are semi-functional, the $j_{\tau}$-th "key" is nominal semi-functional, and the rest of "keys" are normal. In $\operatorname{Game}_{j_{\tau}}^{T}$ the first $(j-1)_{\tau}$ "keys" are semi-functional, the $j_{\tau}$-th "key" is temporary semi-functional, and the remaining "keys" are normal.

To transform $\operatorname{Game}_{(j-1)_{\tau}}$ (where $j_{\tau}$-th "key" is normal) to Game $j_{\tau}$ (where $j_{\tau}$-th "key" is semi-functional), we first convert $\operatorname{Game}_{(j-1)_{\tau}}$ to $\operatorname{Game}_{j_{\tau}}^{N}$, then to $\operatorname{Game}_{j_{\tau}}^{T}$, and finally to Game $j_{j_{\tau}}$. To get from Game To $_{j_{\tau}}^{N}$ to $\operatorname{Game}_{j_{\tau}}^{T}$, we treat the simulations for the queries of Phase 1 and that of Phase 2 differently: the former is based on the three party Diffie-Hellman assumption, and the latter is based on the source group $q$-parallel BDHE assumption. In Game $_{q}=$ Game $_{q_{d e c}}$ all "keys" are semi-functional, and the challenge ciphertext is semi-functional for one of the given messages. Game $_{\text {final }}$ is the final game where all "keys" are semi-functional and the challenge ciphertext is semi-functional for a random message, independent of the two message given by $\mathcal{A}$. We will prove the above games to be indistinguishable by the following lemmas. Note we implicitly assume $S Y M$ is a CCA-secure one-time symmetric encryption, $O T S$ is a strongly existential unforgeable one-time signature, $T C R_{1}, T C R_{2}$ are TCR hash functions and it is hard to find a non-trivial factor of $N$ (for Lemma 3 and Lemma 4).

Lemma 1. If there is an algorithm $\mathcal{A}$ such that Game $_{\text {real }} A d v_{\mathcal{A}}^{C P-A B P R E}-$ Game $_{0} A d v_{\mathcal{A}}^{C P-A B P R E}=\varphi$, we build an algorithm $\mathcal{C}$ that breaks the general subgroup decision assumption with advantage $\varphi$.

Lemma 2. If there is an algorithm $\mathcal{A}$ such that $\operatorname{Game}_{(j-1)_{\tau}} A d v_{\mathcal{A}}^{C P-A B P R E}$ - Game $_{j_{\tau}}^{N} A d v_{\mathcal{A}}^{C P-A B P R E}=\varphi$ (for any $j_{\tau} \in\left[1, q_{\tau}\right]$ ), we build an algorithm $\mathcal{C}$ that breaks the general subgroup decision assumption with advantage $\varphi$. 
Lemma 3. If there is an algorithm $\mathcal{A}$ such that $\operatorname{Game}_{j_{\tau}}^{N} A d v_{\mathcal{A}}^{C P-A B P R E}$ - Game $_{j_{\tau}}^{T} A d v_{\mathcal{A}}^{C P-A B P R E}=\varphi$ for a $j_{\tau}$ belonging to the Phase 1 queries, we build an algorithm $\mathcal{C}$ that breaks the three party Diffie-Hellman assumption in a subgroup with advantage $\varphi$.

Lemma 4. If there is an algorithm $\mathcal{A}$ such that $\operatorname{Game}_{j_{\tau}}^{N} A d v_{\mathcal{A}}^{C P-A B P R E}$ - Game $_{j_{\tau}}^{T} A d v_{\mathcal{A}}^{C P-A B P R E}=\varphi$ for a $j_{\tau}$ belonging to the Phase 2 queries, we build an algorithm $\mathcal{C}$ that breaks the source group q-parallel BDHE assumption in a subgroup with advantage $\varphi$.

Lemma 5. If there is an algorithm $\mathcal{A}$ such that $\operatorname{Game}_{j_{\tau}}^{T} A d v_{\mathcal{A}}^{C P-A B P R E}$ - Game $_{j_{\tau}} A d v_{\mathcal{A}}^{C P-A B P R E}=\varphi$ (for any $j_{\tau} \in\left[1, q_{\tau}\right]$ ), we build an algorithm $\mathcal{C}$ that breaks the general subgroup decision assumption with advantage $\varphi$.

Lemma 6. If there is an algorithm $\mathcal{A}$ such that Game $_{q} A d v_{\mathcal{A}}^{C P-A B P R E}$ - Game $_{\text {final }} A d v_{\mathcal{A}}^{C P-A B P R E}=\varphi$, we can build a reduction algorithm $\mathcal{C}$ that breaks Assumption 1 with advantage $\varphi$.

Due to limited space, we will provide the proofs of the lemmas in the full version of this paper.

\section{Conclusions}

This paper defined the IND-CCA security notion for CP-ABPRE systems, and proposed the first adaptively CCA-secure CP-ABPRE scheme without loss of expressiveness on access policy by integrating the dual system encryption technology with selective proof technique. Following the proof framework introduced by Lewko and Waters, our scheme was proved in the standard model. This paper also motivates interesting open problems, such as, converting our system in the prime order bilinear group.

\section{References}

1. G. Ateniese, K. Fu, M. Green, and S. Hohenberger. Improved proxy re-encryption schemes with applications to secure distributed storage. ACM Trans. Inf. Syst. Secur., 9(1):1-30, 2006.

2. N. Attrapadung, J. Herranz, F. Laguillaumie, B. Libert, E. de Panafieu, and C. Rafols. Attribute-based encryption schemes with constant-size ciphertexts. Theoretical Computer Science, 422(0):15-38, 2012.

3. M. Bellare and S. Shoup. Two-tier signatures, strongly unforgeable signatures, and fiat-shamir without random oracles. In T. Okamoto and X. Wang, editors, Public Key Cryptography, volume 4450 of LNCS, pages 201-216. Springer, 2007. 
4. J. Bethencourt, A. Sahai, and B. Waters. Ciphertext-policy attribute-based encryption. In IEEE Symposium on Security and Privacy, pages 321-334. IEEE Computer Society, 2007.

5. M. Blaze, G. Bleumer, and M. Strauss. Divertible protocols and atomic proxy cryptography. In K. Nyberg, editor, EUROCRYPT, volume 1403 of LNCS, pages 127-144. Springer, 1998.

6. R. Canetti and S. Hohenberger. Chosen-ciphertext secure proxy re-encryption. In P. Ning, S. D. C. di Vimercati, and P. F. Syverson, editors, ACM Conference on Computer and Communications Security, pages 185-194. ACM, 2007.

7. L. Cheung and C. C. Newport. Provably secure ciphertext policy ABE. In P. Ning, S. D. C. di Vimercati, and P. F. Syverson, editors, ACM Conference on Computer and Communications Security, pages 456-465. ACM, 2007.

8. R. Cramer and V. Shoup. Design and analysis of practical public-key encryption schemes secure against adaptive chosen ciphertext attack. SIAM J. Comput., 33(1):167-226, January 2004.

9. V. Goyal, A. Jain, O. Pandey, and A. Sahai. Bounded ciphertext policy attribute based encryption. In L. Aceto, I. Damgård, L. A. Goldberg, M. M. Halldórsson, A. Ingólfsdóttir, and I. Walukiewicz, editors, ICALP (2), volume 5126 of LNCS, pages 579-591. Springer, 2008.

10. V. Goyal, O. Pandey, A. Sahai, and B. Waters. Attribute-based encryption for fine-grained access control of encrypted data. In A. Juels, R. N. Wright, and S. D. C. di Vimercati, editors, ACM Conference on Computer and Communications Security, pages 89-98. ACM, 2006.

11. G. Hanaoka, Y. Kawai, N. Kunihiro, T. Matsuda, J. Weng, R. Zhang, and Y. Zhao. Generic construction of chosen ciphertext secure proxy re-encryption. In O. Dunkelman, editor, Topics in Cryptology - CT-RSA 2012, volume 7178 of $L N C S$, pages 349-364. Springer Berlin Heidelberg, 2012.

12. T. Isshiki, M. H. Nguyen, and K. Tanaka. Proxy re-encryption in a stronger security model extended from ct-rsa2012. In CT-RSA 2012, volume 7779 of $L N C S$, pages 277-292. Springer, 2013.

13. A. B. Lewko, T. Okamoto, A. Sahai, K. Takashima, and B. Waters. Fully secure functional encryption: Attribute-based encryption and (hierarchical) inner product encryption. In H. Gilbert, editor, EUROCRYPT, volume 6110 of $L N C S$, pages 62 91. Springer, 2010.

14. A. B. Lewko and B. Waters. New proof methods for attribute-based encryption: Achieving full security through selective techniques. In R. Safavi-Naini and R. Canetti, editors, CRYPTO, volume 7417 of LNCS, pages 180-198. Springer, 2012.

15. K. Liang, L. Fang, W. Susilo, and D. S. Wong. A ciphertext-policy attribute-based proxy re-encryption with chosen-ciphertext security. In INCoS, pages 552-559. IEEE, 2013.

16. X. Liang, Z. Cao, H. Lin, and J. Shao. Attribute based proxy re-encryption with delegating capabilities. In W. Li, W. Susilo, U. K. Tupakula, R. Safavi-Naini, and V. Varadharajan, editors, ASIACCS, pages 276-286. ACM, 2009.

17. B. Libert and D. Vergnaud. Unidirectional chosen-ciphertext secure proxy reencryption. In R. Cramer, editor, Public Key Cryptography, volume 4939 of LNCS, pages 360-379. Springer, 2008.

18. S. Luo, J. bin $\mathrm{Hu}$, and Z. Chen. Ciphertext policy attribute-based proxy reencryption. In M. Soriano, S. Qing, and J. López, editors, ICICS, volume 6476 of $L N C S$, pages 401-415. Springer, 2010. 
19. M. Mambo and E. Okamoto. Proxy cryptosystems: Delegation of the power to decrypt ciphertexts. IEICE Transactions, E80-A(1):54-63, 1997.

20. T. Mizuno and H. Doi. Hybrid proxy re-encryption scheme for attribute-based encryption. In F. Bao, M. Yung, D. Lin, and J. Jing, editors, Information Security and Cryptology, volume 6151 of LNCS, pages 288-302. Springer Berlin Heidelberg, 2011.

21. A. Sahai and B. Waters. Fuzzy identity-based encryption. In R. Cramer, editor, Advances in Cryptology EUROCRYPT 2005, volume 3494 of LNCS, pages 457473. Springer Berlin Heidelberg, 2005.

22. B. Waters. Ciphertext-policy attribute-based encryption: An expressive, efficient, and provably secure realization. In D. Catalano, N. Fazio, R. Gennaro, and A. Nicolosi, editors, Public Key Cryptography, volume 6571 of LNCS, pages 53-70. Springer, 2011.

23. B. Waters. Functional encryption for regular languages. In R. Safavi-Naini and R. Canetti, editors, CRYPTO, volume 7417 of LNCS, pages 218-235. Springer, 2012.

24. J. Weng, M. Chen, Y. Yang, R. H. Deng, K. Chen, and F. Bao. CCA-secure unidirectional proxy re-encryption in the adaptive corruption model without random oracles. Science China Information Sciences, 53(3):593-606, 2010.

25. J. Weng, Y. Yang, Q. Tang, R. H. Deng, and F. Bao. Efficient conditional proxy re-encryption with chosen-ciphertext security. In ISC '09, volume 5735 of LNCS, pages 151-166. Springer, 2009.

26. J. Weng, Y. Zhao, and G. Hanaoka. On the security of a bidirectional proxy reencryption scheme from PKC 2010. In $P K C$ '11, volume 6571 of $L N C S$, pages 284-295. Springer, 2011.

\section{A Preliminaries}

Due to limited space, we refer the reader to [14] for the definition of composite order bilinear groups, assumption 1, the general subgroup decision assumption, the three party Diffie-Hellman assumption in a subgroup, the source group q-parallel BDHE assumption in a subgroup. We here review the one-time symmetric encryption system.

One-time Symmetric Encryption. A one-time symmetric encryption [8] consists of the following algorithms. Note let $\mathcal{K}_{D}$ be the key space $\{0,1\}^{\text {poly }\left(1^{k}\right)}$, and $S Y M$ be a symmetric encryption scheme, where $\operatorname{poly}\left(1^{k}\right)$ is the fixed polynomial size (bound) with respect to the security parameter $k$. The encryption algorithm $S Y M$.Enc intakes a key $K \in \mathcal{K}_{D}$ and a message $M$, outputs a ciphertext $C$. The decryption algorithm $S Y M . D e c$ intakes $K$ and $C$, outputs $M$ or a symbol $\perp$. The CCA security model for $S Y M$ systems is given in [12], we hence omit the details. 